\title{
Azithromycin effectively inhibits tumor angiogenesis by suppressing vascular endothelial growth factor receptor 2-mediated signaling pathways in lung cancer
}

\author{
FAJIU LI, JIE HUANG, DONGYUAN JI, QINGHUA MENG, CHUANHAI WANG, SHI CHEN, \\ XIAOJIANG WANG, ZHIYANG ZHU, CHENG JIANG, YI SHI, SHUANG LIU and CHENGHONG LI \\ Department of Respiratory Medicine, Wuhan No. 6 Hospital, \\ Affiliated Hospital to Jianghan University, Wuhan, Hubei 430072, P.R. China
}

Received February 6, 2016; Accepted March 3, 2017

DOI: $10.3892 / 01.2017 .6103$

\begin{abstract}
Tumor angiogenesis is essential during lung cancer development and targeting angiogenesis may possess a potential therapeutic value. The present study demonstrates that azithromycin, a Food and Drug Administration-approved antibiotic drug, is a novel tumor angiogenesis inhibitor. Azithromycin inhibits capillary network formation of human lung tumor associated-endothelial cells (HLT-ECs) in vitro and in vivo. It significantly inhibits HLT-EC adhesion and vascular endothelial growth factor (VEGF)-induced proliferation of HLT-ECs in a dose-dependent manner without affecting migration. In addition, azithromycin induces apoptosis of HLT-ECs even in the presence of VEGF. Notably, azithromycin inhibits proliferation and induces apoptosis in multiple lung cancer cell lines to a significantly reduced extent compared with in HLT-ECs, suggesting that HLT-ECs are more susceptible to azithromycin treatment. In a lung tumor xenograft model, azithromycin significantly inhibits tumor growth and its anti-tumor activities are achieved by suppressing angiogenesis. Notably, the inhibitory effects of azithromycin on angiogenesis are associated with its ability to suppress VEGF-induced activation of VEGF receptor 2 (VEGFR2), phosphoinositide 3-kinase (PI3K)/protein kinase B (Akt), focal adhesion kinase, and disruption of focal adhesion assembly and actin stress fiber formation in HLT-ECs. The present study identifies that azithromycin targets VEGFR2-mediated focal adhesion and PI3K/Akt signaling pathways in HLT-ECs, leading to the suppression of angiogenesis and lung tumor growth.
\end{abstract}

Correspondence to: Professor Chenghong Li, Department of Respiratory Medicine, Wuhan No. 6 Hospital, Affiliated Hospital to Jianghan University, 168 Xianggang Road, Wuhan, Hubei 430072, P.R. China

E-mail: drchenghong.li@hotmail.com

Key words: azithromycin, tumor angiogenesis, lung cancer, vascular endothelial growth factor/vascular endothelial growth factor receptor 2 signaling, focal adhesion, phosphoinositide 3-kinase/Akt

\section{Introduction}

Lung cancer is one of the leading causes of cancer associated mortality worldwide, with a 5-year survival rate of $20 \%$ (1). The conventional treatment of cytotoxic chemotherapy for lung cancer appears to have reached an effectiveness plateau and new treatment options are required $(2,3)$. Angiogenesis, the growth of new blood vessels from pre-existing ones, is a fundamental step in the transition of tumors between the dormant and malignant stages $(4,5)$. Vascular endothelial growth factor (VEGF), through activation of VEGF receptor 1 (VEGFR1) and 2 (VEGFR2), is a potent pro-angiogenic factor and a key mediator of angiogenesis in malignant tissues (6). Previous studies have revealed that increased levels of VEGF and high microvessel density are associated with poor prognosis in lung cancer patients $(7,8)$. In addition, the anti-VEGF monoclonal antibody bevacizumab has been tested to improve tumor response and progression-free survival in patients with non-small cell lung cancer (NSCLC) (3,9). Tumor angiogenesis has been the target of major drug discovery programs and angiogenesis inhibitors have been evaluated in clinical trials for lung cancer treatment for the last decade (2,3,10-12).

Azithromycin is a Food and Drug Administration-approved antibiotic and the primary drug for the treatment of chlamydia and mycoplasma pneumonia (13). It destroys bacteria by inhibiting protein synthesis through reversible binding to the 50S subunit of bacterial ribosomes (14). With the exception of its antimicrobial activity, azithromycin exerts anti-inflammatory effects by suppressing the secretion of pro-inflammatory cytokines, including interluekin-8 (15). Previously, azithromycin was reported to inhibit the proliferation of cervical and gastric tumor cells $(16,17)$ and enhance the effects of chemotherapy in NSCLC, however little is known about the underlying mechanisms of its anti-cancer activities (18).

In the present study, the effect of azithromycin in lung tumor angiogenesis was investigated using in vitro and in vivo angiogenesis models, and a lung tumor xenograft model. The present results demonstrated that azithromycin potently inhibits angiogenesis in vitro and in vivo by inducing apoptosis and inhibiting the spreading and proliferation of human lung 
tumor associated-endothelial cells (HLT-ECs). Azithromycin also effectively inhibits lung tumor growth by suppressing angiogenesis. Finally, the inhibitory effects of azithromycin on angiogenesis are identified to be attributed to its inhibition of VEGFR2-mediated downstream signaling pathways.

\section{Materials and methods}

HLT-EC, reagents and drugs. Primary HLT-EC was purchased from Cell Biologics, Inc. (Chicago, IL, USA) and grown in Complete Human Endothelial Cell Medium (Cell Systems Corporation, Kirkland, WA, USA). HLT-EC used for the experiments were from passages 2-4 and starved in endothelial cell medium (ECM, served as the basal medium; Cell Systems Corporation) for $3 \mathrm{~h}$ prior to being treated with drugs. Human lung cancer PC-9, A549, NCI-H69, DMS-53, H157 and EBC-1 cell lines, were purchased from American Type Culture Collection (Manassas, VA, USA) and cultured in Dulbecco's modified Eagle's medium (DMEM; Thermo Fisher Scientific, Inc., Waltham, MA, USA) containing $10 \%$ fetal bovine serum (HyClone; GE Healthcare Life Sciences, Logan, UT, USA), $1 \mathrm{mM}$ sodium pyruvate, $2 \mathrm{mM}$ L-glutamine and $1 \%$ penicillin-streptomycin (Thermo Fisher Scientific, Inc.). Recombinant human $\mathrm{VEGF}_{165}$ was purchased from R\&D Systems, Inc. (Minneapolis, MN, USA). Azithromycin (Sigma-Aldrich; EMD Millipore, Billerica, MA, USA) was dissolved in dimethyl sulfoxide.

In vitro capillary networkformation. The HLT-ECs (2x104/well), together with various concentrations of azithromycin, were seeded onto solidified Matrigel matrix (BD Biosciences, Franklin Lakes, CA, USA) in a 96-well plate. Following 6 h of incubation at $37^{\circ} \mathrm{C}$, capillary network formation was analyzed under light microscopy (Zeiss GmbH, Jena, Germany). The total tube length was quantified using NIH Image J 1.32 software (National Institutes of Health, Bethesda, MD, USA).

Transwell migration assay. A Transwell migration assay (Cell Biolabs, Inc., San Diego, CA, USA) was performed using $6.5 \mathrm{~mm}$ diameter polycarbonate filters pre-coated with $0.1 \%$ gelatin. The HLT-ECs $\left(4 \times 10^{4} /\right.$ well) were seeded into the upper chambers of the Transwell plates, and subsequently treated with azithromycin. ECM with or without $20 \mathrm{ng} / \mathrm{ml}$ VEGF was placed into the lower chamber. Following 4-6 h of incubation at $37^{\circ} \mathrm{C}$, cells spreading on the upper surfaces of the filter (non-migrated cells) were wiped away with cotton swabs, and the migrated cells on the lower surface of the filter were fixed and stained with $0.4 \%$ Giemsa (Sigma-Aldrich; EMD Millipore).

Cell spreading assay. HLT-ECs together with various concentrations $(1,5$ and $10 \mu \mathrm{M})$ of azithromycin were seeded onto a 20X diluted Matrigel-coated 96-well-plate for $2 \mathrm{~h}$. The attached cells were then photographed under light microscopy. Images were taken using magnification x 20 under phase-contrast.

Measurement of proliferation and apoptosis. HLT-ECs were treated with azithromycin in ECM medium with or without $20 \mathrm{ng} / \mathrm{ml}$ VEGF. Lung cancer cells were treated with azithromycin in DMEM medium. Following 2 days of treatment, cellular proliferation activity was measured by the CellTiter $96^{\circledR}$ aqueous one solution cell proliferation assay kit (Promega Corporation, Madison, MI, USA). To measure cell apoptosis, cells were stained with Annexin V-fluorescein isothiocyanate (FITC) and then analyzed on a Beckman Coulter FC500 flow cytometer. The percentage of Annexin V-positive cells was determined by CXP software analysis (Beckman Coulter, Inc., Brea, CA, USA).

Denaturing SDS-PAGE and western blot (WB) analysis. HLT-ECs were treated with azithromycin in endothelial cell medium with or without $20 \mathrm{ng} / \mathrm{ml} \mathrm{VEGF} \mathrm{for} 30 \mathrm{~min}$ and subsequently lysed using a radioimmunoprecipitation assay (RIPA) buffer (Thermo Fisher Scientific, Inc.) to extract total protein. Frozen tumor tissues were homogenized using a polytron homogenizer in ice-cold RIPA buffer for $10 \mathrm{~min}$. Equal amounts $(10 \mu \mathrm{g})$ of protein from cell extracts or tumor tissues were resolved using denaturing 10-15\% SDS-PAGE and analyzed by WB analysis. Antibodies (dilution, 1:1,000) used in WB analyses included anti-p-VEGFR2 (no. 2478), anti-VEGFR2 (no. 2479), anti-VEGF (no. 2463), anti-hypoxia-inducible factor (HIF; no. 3716), anti-phosphorylated (p)-focal adhesion kinase (FAK; no. 3283), anti-FAK (no. 3285), anti-p-phosphatidylinositol 3-kinase (PI3K; no. 4228), anti-PI3K (no. 4292), anti-p-protein kinase B (Akt; no. 4060), anti-Akt (no. 9272) and anti-actin (no. 4967; all from Cell Signaling Technology, Inc., Danvers, MA, USA). The membranes were incubated with primary antibodies at $4^{\circ} \mathrm{C}$ overnight.

Directed in vivo angiogenesis assay. The in vivo angiogenesis in Matrigel plug was determined using the directed in vivo angiogenesis assay kit (Trevigen, Inc., Gaithersburg, MD, USA) (19). The angioreactors were filled with basement membrane extracts alone, in combination with VEGF (20 ng/ml)/basic fibroblast growth factor (bFGF; $30 \mathrm{ng} / \mathrm{ml}$ ) or with VEGF/bFGF in combination with 1,5 or $10 \mu \mathrm{M}$ azithromycin, and incubated at $37^{\circ} \mathrm{C}$ for $1 \mathrm{~h}$ to allow gelling. The angioreactors containing total $20 \mu \mathrm{l}$ volume were implanted under the skin of right flank of severe combined immunodeficiency mice for two weeks. All work was conducted with the formal approval of the Jianghan University Animal Care Committee (Jianghan, China) and strictly followed the ethical guidelines on the care and use of animals. Male SCID mice with 20-25 g were purchased from Animal Resources Centre Australia (Murdoch, WA, Australia) and housed in specific pathogen-free rooms in animal holding unit of Jianghan University. A maximum of 5 mice were maintained in a cage with water bottle and overhead food hopper. A total of 25 mice at 6 -week old were used. The invaded endothelial cells were isolated and labeled with FITC-lectin. The quantification of FITC-lectin labeled endothelial cells was performed using a SPECTRAmax microplate spectrofluorometer (excitation $485 \mathrm{~nm}$ and emission $510 \mathrm{~nm}$; Molecular Devices, LLC, Sunnyvale, CA, USA).

Lung xenograft mouse tumor in SCID mice and blood vessel analysis. All procedures were conducted according to the guidelines approved by the Institutional Animal Care and Use Committee. SCID mice at 6 weeks old were purchased from Animal Resources Centre Australia (Murdoch, WA, Australia). In total 20 male mice (20-25 g) were used in the present study. Mice were housed in pathogen-free room with 


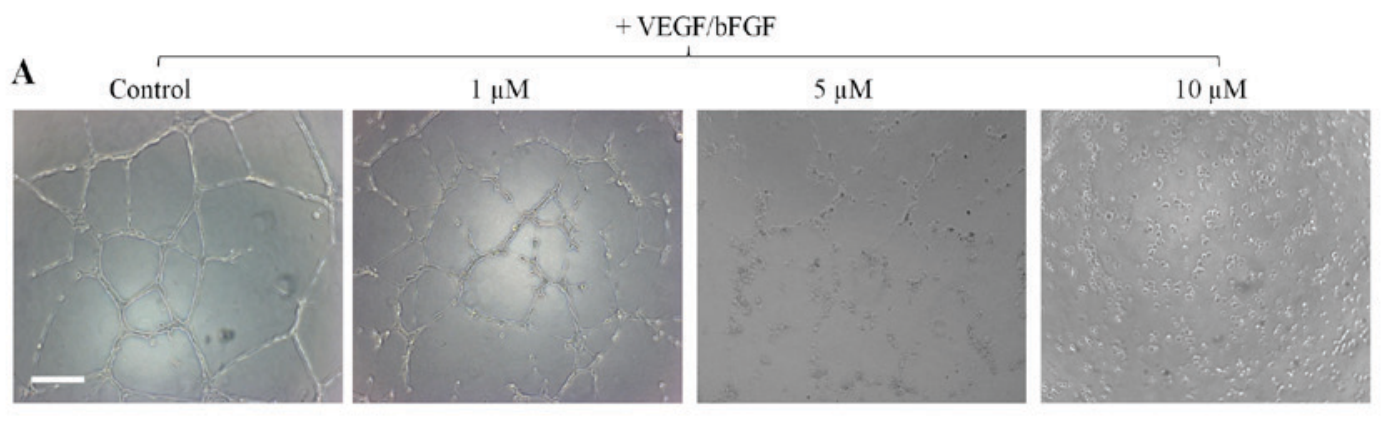

B

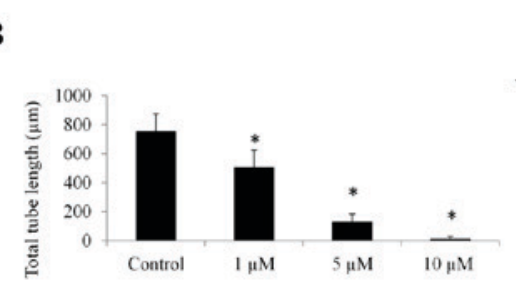

C

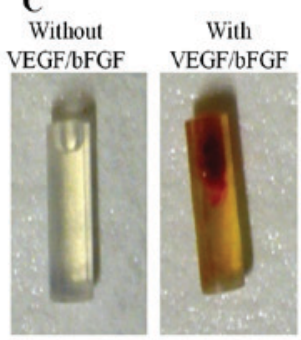

+ VEGF/bFGF

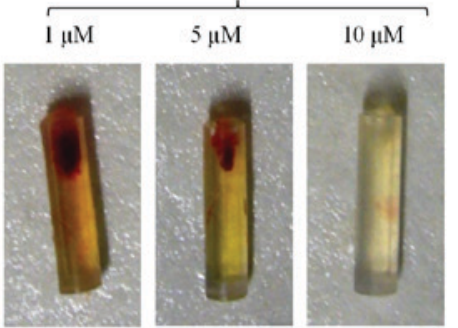

D

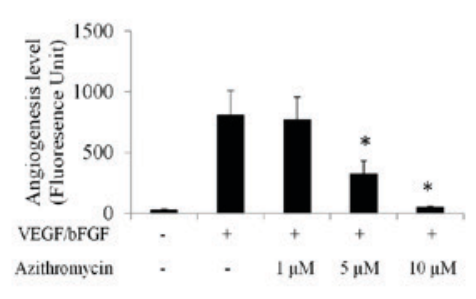

Figure 1. Azithromycin effectively inhibits lung tumor angiogenesis in vitro and in vivo. (A) Representative images of an in vitro capillary network formation were captured. HTL-ECs together with drugs were plated onto complete Matrigel matrix and capillary networks were formed within 6 h. Magnification, x50. Scale bar represents $200 \mu \mathrm{m}$. (B) Quantification of capillary tube length. Azithromycin significantly inhibits in vitro capillary network formation of HLT-ECs in a dose-dependent manner. (C) Azithromycin potently inhibits VEGF/bFGF induced angiogenesis in vivo. Representative images taken 14 days into an in vivo angiogenesis assay. A mixture of $30 \mathrm{ng} / \mu \mathrm{l}$ of bFGF and $20 \mathrm{ng} / \mu \mathrm{l}$ of VEGF was used. (D) Quantification of angiogenesis using fluorescein isothiocyanate-lectin. These data are derived from three independent experiments. ${ }^{*} \mathrm{P}<0.05$, compared with the control or VEGF/bFGF group. HTL-ECs, human lung tumor-associated endothelial cells; VEGF, vascular endothelial growth factor; bFGF, fibroblast growth factor-basic.

temperature $\sim 25^{\circ} \mathrm{C}$. A maximum of 5 were maintained in a cage with water bottle and overhead food hopper. A $14 / 10 \mathrm{~h}$ light/dark cycle is used. Lung tumor A549 cells at 1x106/100 $\mu 1$ PBS were injected into the flank of each mouse. When the tumor volume reached $\sim 200 \mathrm{~mm}^{3}$, the mice were treated with vehicle control $(20 / 80 \%$, DMSO/Saline) or intraperitoneal azithromycin at $20 \mathrm{mg} / \mathrm{kg}$ daily ( $\mathrm{n}=10$ per group). Tumor length and width was measured every three days and the volume was calculated as: Length $\mathrm{x}$ width ${ }^{2} \mathrm{x} 0.5$. For tumor blood vessel analysis, tumor frozen section slides $(5 \mu \mathrm{m})$ were fixed with $4 \%$ paraformaldehyde (Sigma-Aldrich; EMD Millipore, Billerica, MA, USA) and stained with 1:200-diluted anti-CD31 antibody (no MA3100) followed by 1:500-diluted Alex-fluor 488-conjugated 2nd antibody (no A-11034; Molecular Probes; Thermo Fisher Scientific, Inc.). The nuclei were stained with 4, 6-DAPI (Molecular Probes; Thermo Fisher Scientific, Inc.). Blood vessels were viewed with x40 magnification under confocal microscopy (Zeiss GmbH). For vessel quantification, the average number of vessels per microscopic field from 3 microscopic fields per tumor section were analyzed.

Analysis of actin stress fibers and focal adhesions in HLT-ECS. HLT-ECs were treated with azithromycin in ECM medium with or without $20 \mathrm{ng} / \mathrm{ml} \mathrm{VEGF}$ for $60 \mathrm{~min}$ at $37^{\circ} \mathrm{C}$. Cells were fixed with $4 \%$ formalin for $10 \mathrm{~min}$ and washed with $1 \mathrm{X}$ PBS. Cells were then permeabilized with $0.5 \%$ Triton X-100 for $10 \mathrm{~min}$ and stained with 1:500 diluted anti-paxillin antibody (no sc-373880) and 1:500 diluted FITC-conjugated immunoglobulin G (no sc-20052; both from Santa Cruz Biotechnolgy, Inc., Dallas, TX, US). Tetramethylrhodamine-conjugated phalloidin (Sigma-Aldrich; EMD Millipore) was used to stain the actin cytoskeleton.
Statistical analysis. All data are expressed as the mean \pm standard deviation to indicate data variability. Statistical analyses were performed using Student's unpaired t-test. A one way analysis of variance was used for comparison in instances of multiple parameters. All statistical analyses were performed using the Graphpad Prism v6.0 software (GraphPad Software, Inc., La Jolla, CA, USA). $\mathrm{P}<0.05$ was considered to indicate a statistically significant difference.

\section{Results}

Azithromycin effectively inhibits lung tumor angiogenesis in vitro and in vivo. To investigate the effects of azithromycin in lung tumor angiogenesis, in vitro and in vivo angiogenesis assays were performed using HLT-ECs isolated from human lung tumors. ECs can rapidly align and form tubular structures within $6 \mathrm{~h}$ when cultured on complete Matrigel matrix, which is rich in extracellular matrix and various growth factors, including VEGF and bFGF (20).

Azithromycin was revealed to effectively inhibit capillary network formation of HLT-ECs on complete Matrigel matrix in a dose-dependent manner (Fig. 1A and B). The effector concentration for half maximal response of azithromycin was as low as $\sim 3 \mu \mathrm{m}$ (Fig. 1B). It was then examined whether azithromycin could inhibit angiogenesis in vivo using a modified basic Matrigel plug angiogenesis assay (19). Basic Matrigel together with drugs were retained in a silicon tube and implanted subcutaneously into the mouse for two weeks. As shown in Fig. 1C, a mixture of VEGF and bFGF induced potent angiogenesis compared with the control (basic Matrigel alone). When azithromycin was added together with VEGF/bFGF, it suppressed $\mathrm{VEGF} / \mathrm{bFGF}$-induced angiogenesis in a dose-dependent 
A

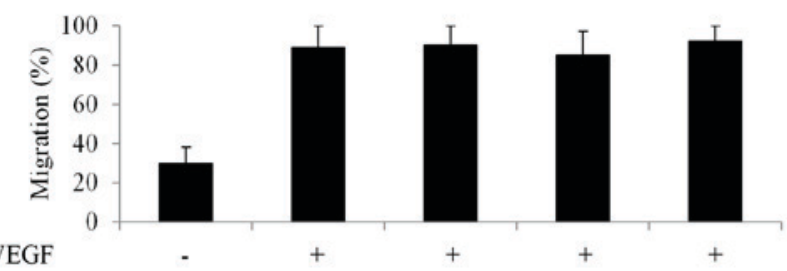

$\begin{array}{llllllllll}\text { Azithromycin } & - & - & 1 \mu \mathrm{M} & 5 \mu \mathrm{M} & 10 \mu \mathrm{M}\end{array}$

B
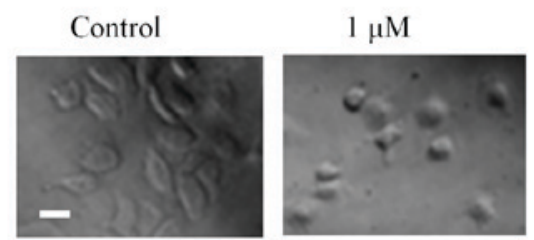

C

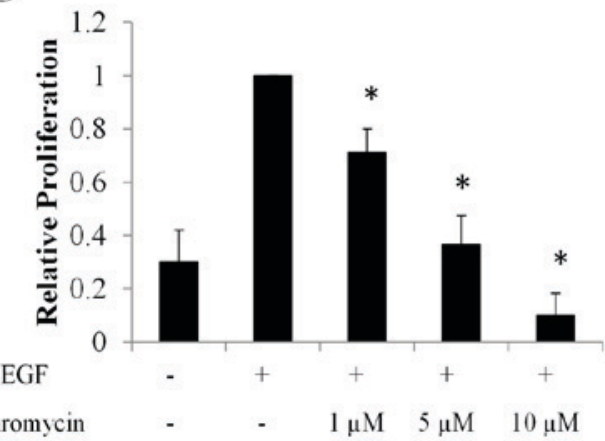

$5 \mu \mathrm{M}$

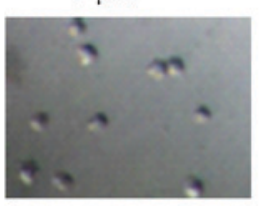

$10 \mu \mathrm{M}$

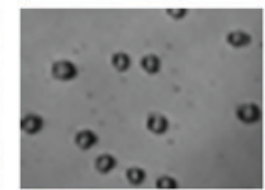

D

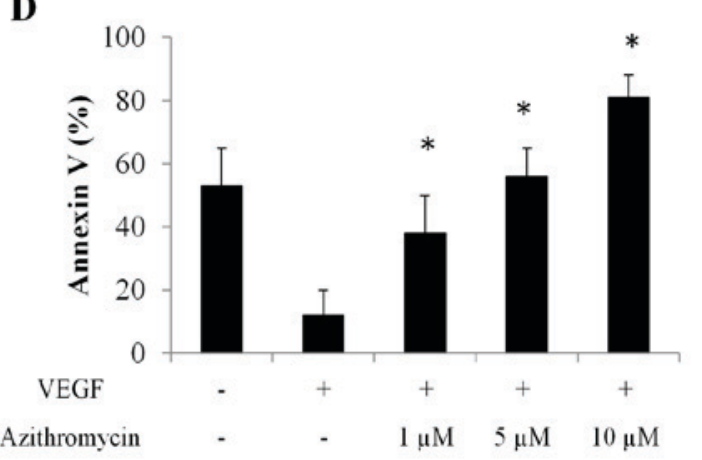

Figure2.EffectsofazithromycinonvariousbiologicalfunctionsofHLT-ECs.(A)AzithromycindoesnotaffectVEGF-inducedHLT-ECsmigration.(B)Azithromycin potently suppresses HLT-EC adhesion and spreading to 10X diluted complete Matrigel. Representative images of a HLT-EC spreading assay were captured. Magnification, x20, scale bar represents $20 \mu \mathrm{m}$. Complete spreading was observed in control cells well-spread in a radial manner and stretched on the surface. (C) Azithromycin inhibits VEGF-stimulated proliferation of HLT-ECs and (D) induces apoptosis of HLT-ECs in the presence of VEGF. VEGF (20 ng/ml) was used in all experiments. These data are derived from four independent experiments. "P $<0.05$, compared with the VEGF/bFGF group. HTL-ECs, human lung tumorassociated endothelial cells; VEGF, vascular endothelial growth factor; bFGF, fibroblast growth factor-basic.

A

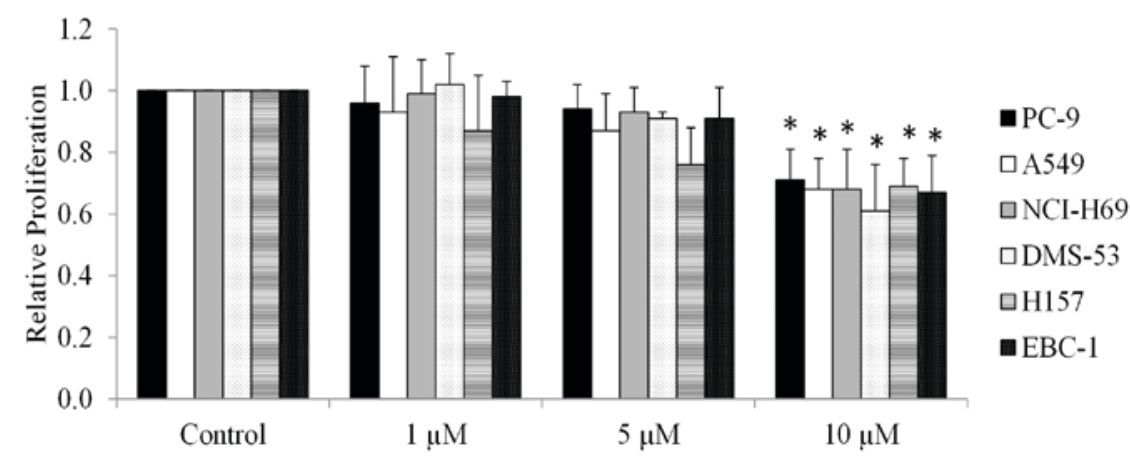

B

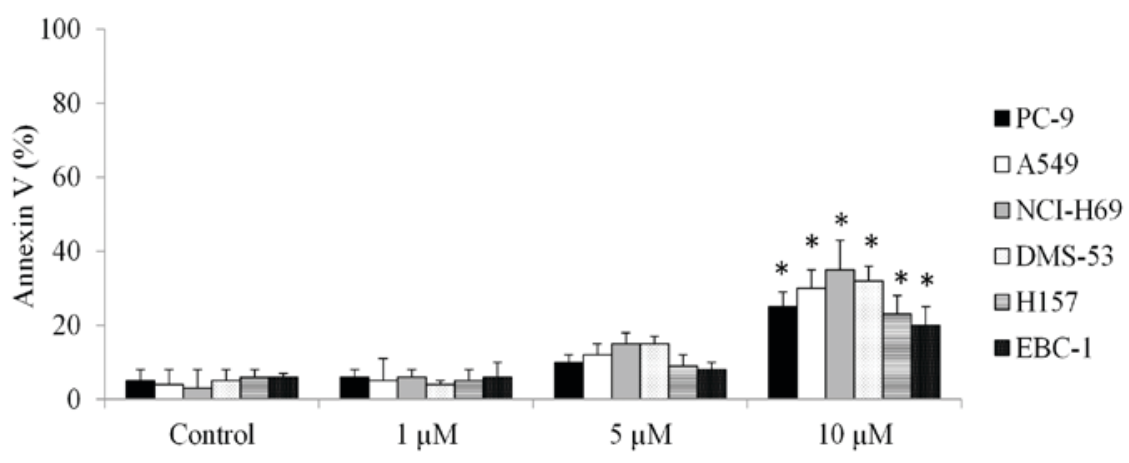

Figure 3. Effects of azithromycin on the proliferation and apoptosis of lung cancer cells. (A) Azithromycin at $10 \mu \mathrm{m}$ but not 1 and $5 \mu \mathrm{m}$ significantly inhibits proliferation and (B) induces apoptosis of multiple lung cancer cell lines, including PC-9, A549, NCI-H69, DMS-53, H157 and EBC-1. DMSO was used as control. ${ }^{*} \mathrm{P}<0.05$, compared with the control. 

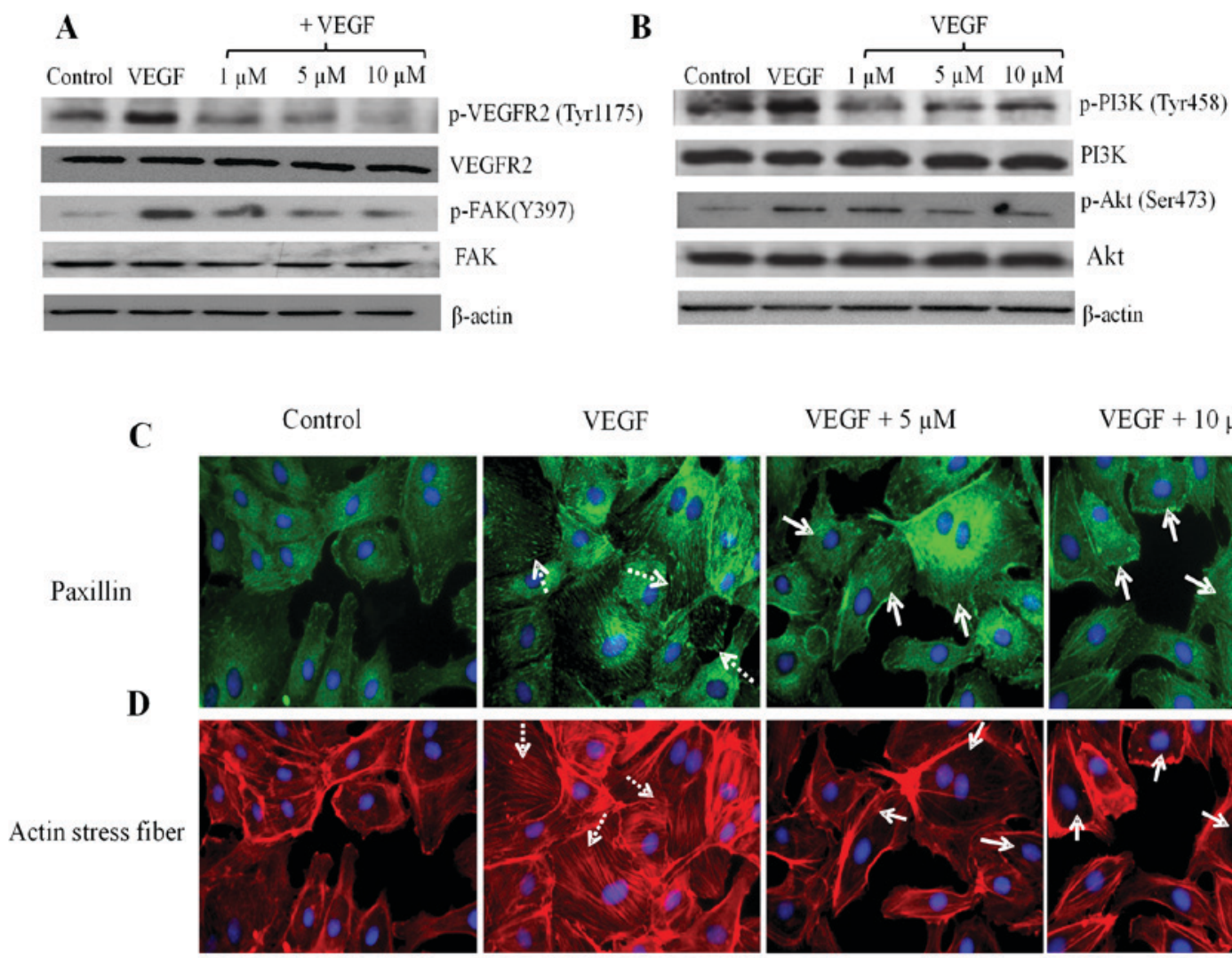

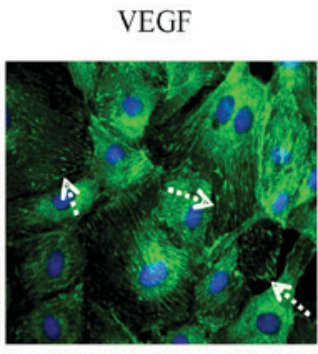

$\mathrm{VEGF}+5 \mu \mathrm{M}$
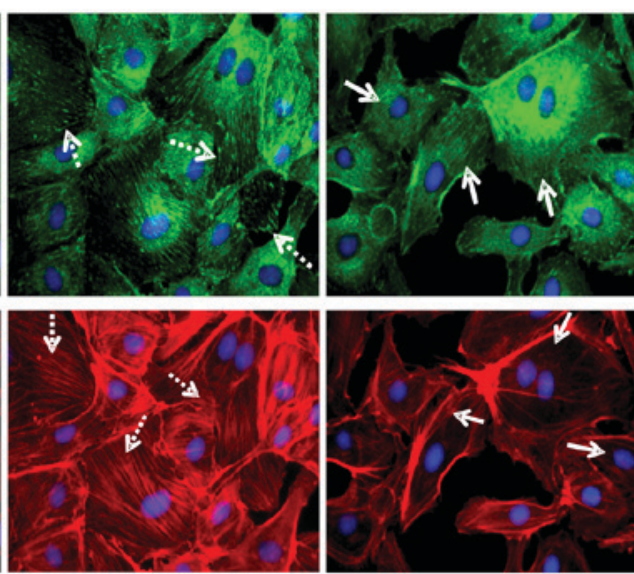

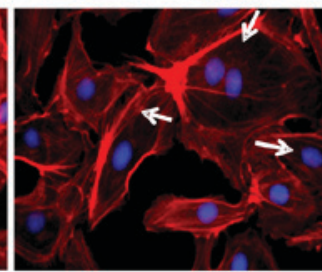

VEGF $+10 \mu \mathrm{M}$
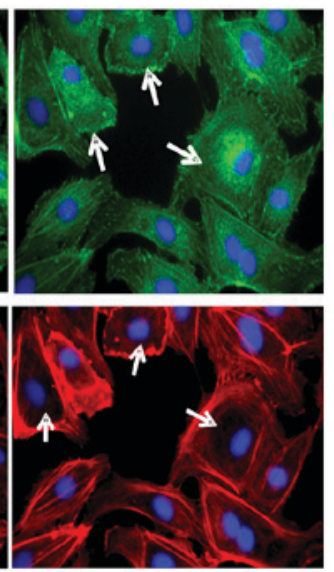

Figure 4. Azithromycin inhibits tumor angiogenesis by suppressing VEGFR2-mediated signaling pathways. Azithromycin suppresses activation of (A) VEGFR2 and (B) its downstream FAK, PI3K/Akt signaling pathways. Western blot analysis of human lung tumor-associated endothelial cells treated with azithromycin for $60 \mathrm{~min}$ in the presence of $20 \mathrm{ng} / \mathrm{ml}$ VEGF. Representative western blot analysis images are shown. $\beta$-actin was used for normalization. (C) Azithromycin inhibits VEGF-stimulated paxillin clustering and recruitment to plasma membrane focal adhesions. Dashed arrows indicate the increased paxillin clustering to plasma membrane, while arrows indicate the reduced paxillin clustering. (D) Azithromycin inhibits VEGF-induced stress fiber formation. Actin stress fibers were visualized with phalloidin (red). Increased stress fibers bundles are indicated by dashed arrows, while reduced stress fiber formation is indicated by arrows. Images shown are representative. Magnification, x400. VEGFR2, vascular endothelial growth factor receptor 2; FAK, focal adhesion kinase; PI3K, phosphoinositide 3-kinase; Akt, protein kinase B; p-, phosphorylated.

manner, as assessed by measuring the number of ECs using fluorescently labeled EC-binding lectin (Fig. 1D). Collectively, the present results demonstrate that azithromycin acts as an angiogenesis inhibitor in vitro and in vivo.

Azithromycin suppresses spreading, proliferation and induces apoptosis of HLT-ECs without affecting HLT-EC migration. Capillary network formation is a multi-step and dynamic process involving cell attachment to matrix, migration, spreading, morphogenesis and apoptosis (21). Therefore, migration, spreading, proliferation and apoptosis assays were performed using HLT-ECs to understand how azithromycin interfered with capillary network formation of lung tumor ECs. VEGF is the most important EC specific angiogenic growth factor, stimulating various aspects of angiogenesis (22); therefore, the effects of azithromycin on VEGF-stimulated angiogenesis were investigated.

Azithromycin was revealed to exhibit no effect on VEGF-induced HLT-ECs migration (Fig. 2A). By contrast, azithromycin significantly inhibited HLT-EC spreading to diluted complete Matrigel [composed of laminin, collage type IV, heparan sulfate proteoglycans and entactin (23); Fig. 2B]. VEGF is known to stimulate proliferation of ECs and protect ECs against apoptosis (22). Results consistently demonstrated that there were more proliferating and less apoptotic HLT-ECs in the presence of VEGF compared with the control (basic medium without VEGF; Fig. 2C and D). Notably, azithromycin inhibited VEGF-induced proliferation of HLT-ECs and induced apoptosis in a dose-dependent manner, even in the presence of VEGF (Fig. 2C and D). Compared with HLT-ECs, azithromycin at the same concentration $(10 \mu \mathrm{m})$ inhibited proliferation and induced apoptosis of multiple lung cancer cell lines, including PC-9, A549, NCI-H69, DMS-53, H157 and EBC-1, to a significantly reduced extent (Fig. 3A and B), suggesting that HLT-ECs were more susceptible compared with lung cancer cells to azithromycin treatment.

Azithromycin inhibits tumor angiogenesis by suppressing vascular endothelial growth factor receptor 2 (VEGFR2) -mediated signaling pathways. To understand the molecular basis of azithromycin-mediated anti-angiogenic effects, the action of azithromycin on the phosphorylation of VEGFR2 was firstly examined, since VEGFR2 is the crucial and main receptor mediating VEGF-stimulated angiogenic activities (24). Azithromycin inhibited VEGFR2 activation by suppressing phosphorylation of VEGFR2 at Tyr1175 at 1,5 and $10 \mu \mathrm{m}$ in HLT-ECs (Fig. 4A), which was consistent with its in vitro and in vivo functions. As a consequence of 


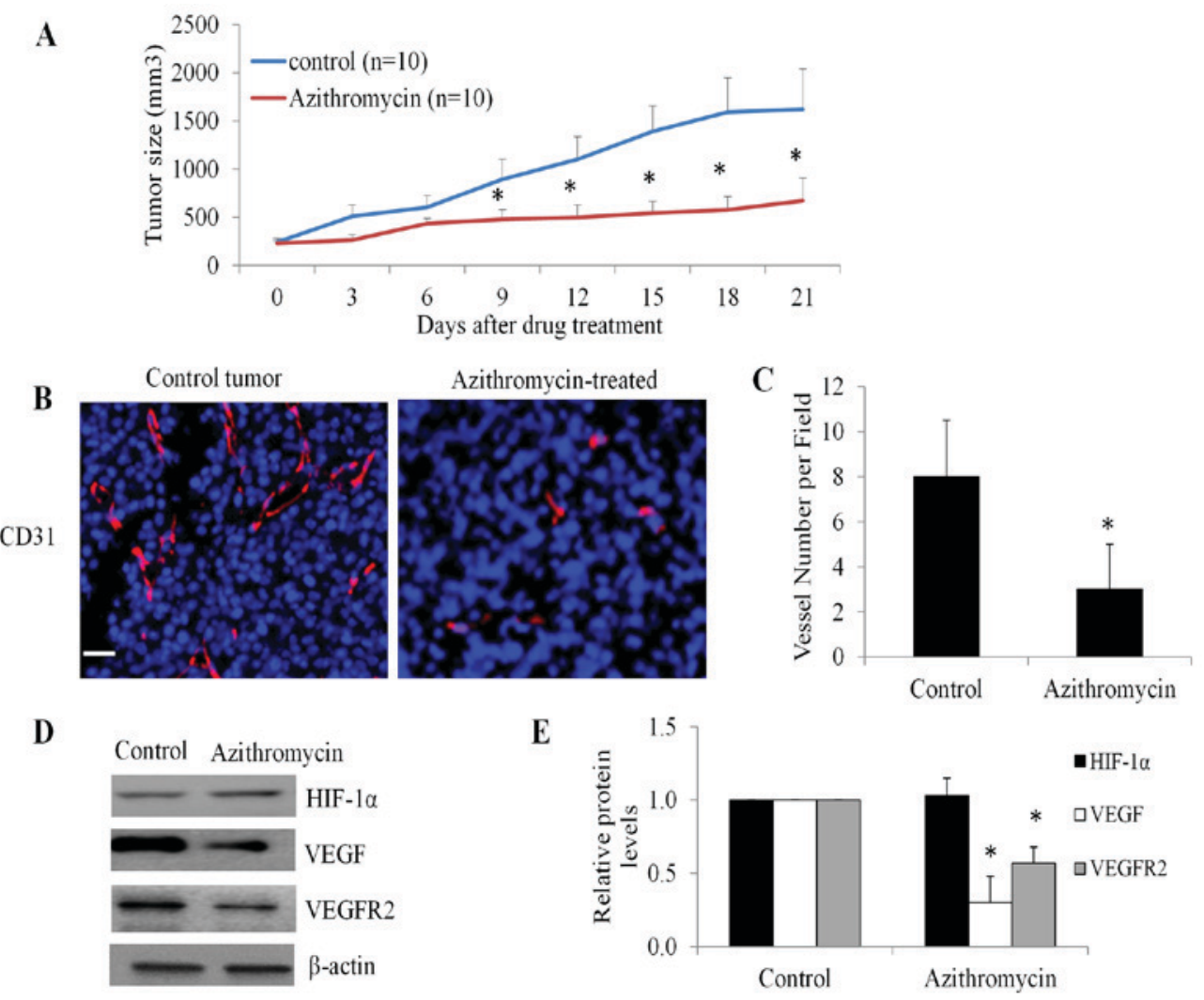

Figure 5. Azithromycin inhibits lung tumor growth and tumor angiogenesis in xenograft mice. (A) Azithromycin significantly inhibits A549 lung tumor growth. SCID mice bearing A549 lung tumor xenografts at the flanks were treated with equal volume of vehicles, azithromycin at 20 mg/kg daily by intraperitoneal injection $(n=10)$. (B) Azithromycin significantly inhibits tumor angiogenesis. Tumor blood vessels were visualized by CD31 staining. Nucleus was stained blue by 4,6-diamidino-2-phenylindole. Scale bar represents $20 \mu \mathrm{m}$. (C) The average number of vessels were analyzed from three microscopic fields per tumor section, three tumor sections per tumor and ten tumors for each experiment group. (D and E) Decreased protein levels of VEGF and VEGFR2, but not HIF-1 $\alpha$, were observed in azithromycin-treated tumors. Representative western blot analysis images of tumor tissues were captured and presented. The protein levels of HIF-1 $\alpha$, VEGF and VEGFR were quantified using Image-J software with $\beta$-actin levels for normalization. "P<0.05, compared with the control. CD31, cluster of differentiation 31; VEGF, vascular endothelial growth factor; VEGFR2, VEGF receptor 2; HIF-1 $\alpha$, hypoxia-inducible factor-1 $\alpha$.

the deactivation of VEGFR2, suppressed phosphorylation of PI3K, Akt and FAK was observed in azithromycin-treated HLT-ECs (Fig. 4A and B).

FAK is an essential component of the focal adhesion complex that attaches cells to the extracellular matrix, and is important for cell spreading and survival (25). Activated FAK then activates and recruits paxillin into the focal adhesion complex, followed by an accumulation of actin stress fibers to form proper focal adhesion complexes. Consistent with inhibition of HLT-EC spreading and FAK activation (Figs. 2B and 4A), azithromycin effectively blocked VEGF-induced paxillin recruitment into focal adhesions, as shown by the reduced paxillin staining (Fig. 4C) and VEGF-induced stress fiber formation (Fig. 4D).

The above experiments associated with each other to reveal that inhibition of HLT-EC spreading, proliferation and survival by azithromycin is linked with its ability to suppress the VEGF-VEGFR2-mediated PI3K/Akt signaling pathway, FAK phosphorylation, paxillin relocation into focal adhesions and actin stress fiber formation.

Azithromycin inhibits lung tumor growth and tumor angiogenesis in xenograft mice. It was then investigated whether azithromycin exerts inhibitory effects on tumor angiogenesis in vivo and tumor growth using a A549 lung tumor xenograft mouse model. The mice tolerated $20 \mathrm{mg} / \mathrm{kg}$ azithromycin well, as no significant body weight loss was observed (data not shown). Azithromycin was revealed to significantly suppressed lung tumor growth (Fig. 5A).

To examine whether the inhibitory effect of azithromycin on lung tumor growth is attributable to decreased tumor angiogenesis, blood vessel density and morphology of tumor tissue sections was examined using anti-CD31 antibody, which specifically stains tumor ECs (26). A significant decrease in density of blood vessels was observed in azithromycin-treated tumors compared with the control (Fig. 5B and C). Notably, the morphology of the majority of blood vessels was non-lumen structures (cell clusters and spots) in azithromycin-treated tumors, whereas blood vessels in control tumors were all luminal structures (Fig. 5B). Consistently, decreased protein levels of VEGF and VEGFR2, but not HIF $\alpha$, were observed in azithromycin-treated tumors (Fig. 5D). These data demonstrated that azithromycin inhibited lung tumor growth through suppressing tumor angiogenesis.

\section{Discussion}

Combination therapies using angiogenesis inhibitors and cytotoxic chemotherapeutic agents may achieve additional anti-tumor efficacy as it targets two aspects of tumor 
development: Tumor cell growth and vascularization. Angiogenesis has been the target of major drug discovery programs, and angiogenesis inhibitors with different mechanisms of actions have been evaluated in clinical trials for cancer treatment over the last decade $(2,3)$. To the best of our knowledge, the present study demonstrated for the first time that azithromycin acts as a potential angiogenesis inhibitor and may be repositioned from its traditional use in infection disease to the therapy of lung cancer.

The primary endothelial cells, HLT-ECs (isolated from human lung tumor), were selected to demonstrate the biological effects of azithromycin on a lung tumor angiogenesis model. Azithromycin was revealed to inhibit capillary network formation of HLT-ECs in a dose-dependent manner (Fig. 1A and B), suggesting that azithromycin inhibits lung tumor angiogenesis in vitro. VEGF is the most important angiogenic factor that stimulates endothelial cell proliferation, migration, tube formation and survival- all necessary components of an angiogenesis response (6). With the exception of migration, azithromycin inhibited all aspects of VEGF-induced angiogenesis, as shown by decreased proliferation and spreading, and increased apoptosis in HLT-ECs (Fig. 2). In accordance with in vitro data, azithromycin potently inhibited $\mathrm{VEGF} / \mathrm{bFGF}$ induced angiogenesis in vivo (Fig. 1C and D). These results demonstrate that azithromycin is an angiogenesis inhibitor, and blocks multiple steps of angiogenesis.

In addition, azithromycin significantly suppressed VEGF-induced activation of VEGFR2, followed by the decreased phosphorylation of PI3K/Akt and FAK, and disruption of focal adhesion assembly and actin stress fiber formation in HLT-ECs (Fig. 4). Inhibitors targeting the PI3K/Akt were shown to decrease VEGF secretion and angiogenesis $(27,28)$. The present study supports previous studies $(27,28)$ and additionally demonstrates that PI3K/Akt may perform essential roles for anti-angiogenic activities of azithromycin. Bloomstein et al (2) reported that VEGF and its main receptor VEGFR2 are two validated molecular targets for patients with NSCLC. The ability of azithromycin to target VEGF and VEGFR2 makes it an attractive addition to the armamentarium in the treatment of NSCLC.

Notably, azithromycin has a potent ability to inhibit human lung tumor growth, an effect achieved to a great extent by angiogenesis suppression. In xenograft lung tumor mice, azithromycin significantly inhibited tumor growth throughout the treatment $(\mathrm{P}=0.023$; Fig. 5A). Vascularization analysis revealed that the generation of new blood vessels and the expression of VEGF and VEGFR2 in azithromycin-treated mice tumors were significantly decreased compared with the control (Fig. 5B-E), demonstrating that azithromycin inhibits angiogenesis in lung tumors. This finding supports the previous studies that angiogenesis inhibitors alone can inhibit tumor growth (29). Notably, azithromycin has been reported to exhibit inhibitory effects on tumor cell proliferation (16). It is possible that the inhibition of lung tumor growth occurs through the effects of azithromycin on the biological function of endothelial cells and/or tumor cells. The present data demonstrated that HLT-ECs were more susceptible compared with lung cancer cells to azithromycin treatment (Fig. 3), suggesting that endothelial cells were possibly the primary target of azithromycin in lung tumor growth inhibition.
In conclusion, to the best of our knowledge, the present study reports for the first time that azithromycin is a novel angiogenesis inhibitor. It potently inhibits angiogenesis in vitro and in vivo through suppressing multiple aspects of VEGF-induced angiogenic response. The molecular mechanisms of the action of azithromycin on HLT-ECs are attributed to its inhibition of VEGFR2-mediated downstream signaling pathways. Notably, azithromycin effectively inhibits lung tumor growth via suppressing angiogenesis. These findings suggest that azithromycin may be translated into clinical trials for lung cancer treatment and also emphasize the therapeutic value of angiogenesis inhibition in lung cancer.

\section{Acknowledgements}

The present study was supported by a research grant provided by Wuhan No. 6 Hospital, Affiliated Hospital to Jianghan University (grant no. WH6201206006) and the National Foundation Research Grant of China (grant no. 81300413).

\section{References}

1. Dancey J and Le Chevalier T: Non-small cell lung cancer: An overview of current management. Eur J Cancer 33 (Suppl 1): S2-S7, 1997.

2. Blumenschein GR Jr, Reckamp K, Stephenson GJ, O'Rourke T, Gladish G, McGreivy J, Sun YN, Ye Y, Parson M and Sandler A: Phase 1b study of motesanib, an oral angiogenesis inhibitor, in combination with carboplatin/paclitaxel and/or panitumumab for the treatment of advanced non-small cell lung cancer. Clin Cancer Res 16: 279-290, 2010.

3. Lind JS and Smit EF: Angiogenesis inhibitors in the treatment of non-small cell lung cancer. Ther Adv Med Oncol 1: 95-107, 2009.

4. Hanahan D and Folkman J: Patterns and emerging mechanisms of the angiogenic switch during tumorigenesis. Cell 86: 353-364, 1996.

5. Folkman J: Tumor angiogenesis: Therapeutic implications. N Engl J Med 285: 1182-1186, 1971.

6. Ferrara N: The role of vascular endothelial growth factor in pathological angiogenesis. Breast Cancer Res Treat 36: 127-137, 1995.

7. Han H, Silverman JF, Santucci TS, Macherey RS, d'Amato TA, Tung MY, Weyant RJ and Landreneau RJ: Vascular endothelial growth factor expression in stage I non-small cell lung cancer correlates with neoangiogenesis and a poor prognosis. Ann Surg Oncol 8: 72-79, 2001.

8. Fontanini G, Bigini D, Vignati S, Basolo F, Mussi A, Lucchi M, Chine S, Angeletti CA, Harris AL and Bevilacqua G: Microvessel count predicts metastatic disease and survival in non-small cell lung cancer. J Pathol 177: 57-63, 1995.

9. Horn L and Sandler AB: Angiogenesis in the treatment of non-small cell lung cancer. Proc Am Thorac Soc 6: 206-217, 2009.

10. Zhao L, Li W, Zhang H, Hou N, Guo L and Gao Q: Angiogenesis inhibitors rechallenge in patients with advanced non-small-cell lung cancer: A pooled analysis of randomized controlled trials. Onco Targets Ther 8: 2775-2781, 2015.

11. Zhang TT, Wang RM, Yang $Z$ and Chen GB: Dual inhibiting EGFR and VEGF pathways versus EGFR-TKIs alone in the treatment of advanced non-small-cell lung cancer: A meta-analysis of randomized controlled trials. Clin Transl Oncol 18: 576-581, 2016.

12. Zhang J, Liu J, Chen H, Wu W, Li X, Wu Y, Zhang K and Gu L: The impact of histological types on the efficacy of angiogenesis inhibitors in the treatment of advanced NSCLC: A meta-analysis of randomized controlled trials. Onco Targets Ther 8: 2375-2382, 2015.

13. Esposito S, Bosis S, Faelli N, Begliatti E, Droghetti R, Tremolati E, Porta A, Blasi F and Principi N: Role of atypical bacteria and azithromycin therapy for children with recurrent respiratory tract infections. Pediatr Infect Dis J 24: 438-444, 2005. 
14. Champney WS and Burdine R: Macrolide antibiotics inhibit 50S ribosomal subunit assembly in Bacillus subtilis and Staphylococcus aureus. Antimicrob Agents Chemother 39: 2141-2144, 1995.

15. Kitsiouli E, Antoniou G, Gotzou H, Karagiannopoulos M Basagiannis D, Christoforidis S, Nakos G and Lekka ME: Effect of azithromycin on the LPS-induced production and secretion of phospholipase A2 in lung cells. Biochim Biophys Acta 1852: $1288-1297,2015$

16. Lamb R, Ozsvari B, Lisanti CL, Tanowitz HB, Howell A Martinez-Outschoorn UE, Sotgia F and Lisanti MP: Antibiotics that target mitochondria effectively eradicate cancer stem cells, across multiple tumor types: Treating cancer like an infectious disease. Oncotarget 6: 4569-4584, 2015

17. Zhou X, Zhang Y, Li Y, Hao X, Liu X and Wang Y: Azithromycin synergistically enhances anti-proliferative activity of vincristine in cervical and gastric cancer cells. Cancers (Basel) 4: 1318-1332, 2012.

18. Chu DJ, Yao DE, Zhuang YF, Hong Y, Zhu XC, Fang ZR, Yu J and Yu ZY: Azithromycin enhances the favorable results of paclitaxel and cisplatin in patients with advanced non-small cell lung cancer. Genet Mol Res 13: 2796-2805, 2014.

19. Guedez L, Rivera AM, Salloum R, Miller ML, Diegmueller JJ, Bungay PM and Stetler-Stevenson WG: Quantitative assessment of angiogenic responses by the directed in vivo angiogenesis assay. Am J Pathol 162: 1431-1439, 2003.

20. Madri JA and Pratt BM: Endothelial cell-matrix interactions: In vitro models of angiogenesis. J Histochem Cytochem 34 85-91, 1986.

21. Davis GE and Senger DR: Endothelial extracellular matrix: Biosynthesis, remodeling, and functions during vascular morphogenesis and neovessel stabilization. Circ Res 97: 1093-1107, 2005.
22. Ferrara N: The role of VEGF in the regulation of physiological and pathological angiogenesis. EXS: 209-231, 2005.

23. Kleinman HK, McGarvey ML, Hassell JR, Star VL, Cannon FB, Laurie GW and Martin GR: Basement membrane complexes with biological activity. Biochemistry 25: 312-318, 1986.

24. Shibuya M: Vascular endothelial growth factor-dependent and -independent regulation of angiogenesis. BMB Rep 41: 278-286, 2008.

25. Lechertier $\mathrm{T}$ and Hodivala-Dilke K: Focal adhesion kinase and tumour angiogenesis. J Pathol 226: 404-412, 2012.

26. Parums DV, Cordell JL, Micklem K, Heryet AR, Gatter KC and Mason DY: JC70: A new monoclonal antibody that detects vascular endothelium associated antigen on routinely processed tissue sections. J Clin Pathol 43: 752-757, 1990.

27. Karar J and Maity A: PI3K/AKT/mTOR pathway in angiogenesis. Front Mol Neurosci 4: 51, 2011

28. Kim GD, Oh J, Park HJ, Bae K and Lee SK: Magnolol inhibits angiogenesis by regulating ROS-mediated apoptosis and the $\mathrm{PI} 3 \mathrm{~K} / \mathrm{AKT} / \mathrm{mTOR}$ signaling pathway in $\mathrm{mES} / \mathrm{EB}$-derived endothelial-like cells. Int J Oncol 43: 600-610, 2013.

29. He L, Wu Y, Lin L, Wang J, Wu Y, Chen Y, Yi Z, Liu M and Pang X: Hispidulin, a small flavonoid molecule, suppresses the angiogenesis and growth of human pancreatic cancer by targeting vascular endothelial growth factor receptor 2-mediated $\mathrm{PI} 3 \mathrm{~K} / \mathrm{Akt} / \mathrm{mTOR}$ signaling pathway. Cancer Sci 102: 219-225, 2011. 